\title{
A FÓRMULA BAROMÉTRICA COMO INSTRUMENTO DE ENSINO EM QUÍMICA
}

Otávio Luiz Bottecchia

Instituto de Química, Universidade Federal de Uberlândia, Av. João Naves de Ávila, 2121, 38400-902 Uberlândia - MG, Brasil

Recebido em 24/9/08; aceito em 5/4/09; publicado na web em 10/8/09

THE BAROMETRIC FORMULA AS RESOURCE FOR TEACHING CHEMISTRY. The barometric equation is revisited. Restrictions imposed for its derivation are investigated. Results are discussed and related to simple themes of ordinary life. The theoretical models fit to experimental data. Correction for temperature effect improves the fitting in comparison to the barometric formula. The scope for application of the model is discussed.

Keywords: barometric formula; atmosphere; model.

\section{INTRODUÇÃO}

Fórmula barométrica ${ }^{1}$ é uma equação matemática que relaciona a pressão de um gás, $P$, em equilíbrio hidrostático na altitude, $h$, com temperatura termodinâmica, $T$, sujeito a um campo gravitacional constante:

$$
P=P_{0} e^{\frac{-M g}{R T} h}
$$

$P_{0}$ é a pressão do gás em um nível de referência com altitude adotada como zero; $g$ é a aceleração da gravidade; $R$ é a constante dos gases; e $M$ é a massa molar do gás.

A dependência da pressão do ar com a altitude era já conhecida no século XVII. Seguindo as idéias de Torricelli, Blaise Pascal, com ajuda de Florin Perrier, constatou a menor pressão atmosférica no alto de uma montanha. ${ }^{2} \mathrm{O}$ crédito da obtenção de uma relação quantitativa e tabular deve-se a Edmund Halley. ${ }^{3}$ A dedução deve-se a Pierre-Simon Laplace ${ }^{4}$. Perrin testou a fórmula barométrica em sistemas coloidais. ${ }^{5}$ Berberan-Santos e colaboradores ${ }^{6}$ discorreram sobre o histórico do processo de descoberta da fórmula barométrica. Martins ${ }^{7}$ analisou o contexto histórico desta época. Ferreira ${ }^{8}$ resgatou as memórias de Francisco José Caldas, que buscou a relação entre temperatura de ebulição e altitude. A referência 9 trata do perfil entrópico de um gás sob ação gravitacional.

O objetivo deste trabalho é mostrar que a fórmula barométrica pode ser explorada no contexto didático. O enfoque é o desenvolvimento de modelos relacionáveis com problemas reais e a ênfase na correlação entre o processo matemático e a realidade física, dirigido ao estudante de graduação.

\section{FUNDAMENTOS TEÓRICOS}

\section{Parte I: A fórmula barométrica (modelo I)}

$$
\text { A pressão define-se por } P=\frac{F}{A} \text {, em que } F \text { é a força aplicada numa }
$$

superfície $A$. Considerando uma coluna vertical com área $A$ e comprimento $l$, portanto, com volume $V=A \times l$, e a força como sendo o peso do gás contido nos limites da coluna, tem-se que $P=\frac{m g}{V} l$.

Reconhecendo a densidade do gás como $\rho=\frac{m}{V}$, obtém-se:

*e-mail: otavio@ufu.br

$$
P=\rho g l
$$

Para medir a pressão atmosférica com um barômetro de mercúrio (ou outro líquido qualquer com densidade conhecida), o comprimento $l$ é a altura da coluna de mercúrio, $H$, em equilíbrio hidrostático. Numa altitude arbitrária de referência, $h_{0}$, que costuma ser adotada como zero, a altura da coluna de mercúrio é $H_{0}$, tal que a pressão atmosférica é $P_{0}=\rho_{H g} g H_{0}$. Numa altitude genérica, $h$, a pressão atmosférica é $P=\rho_{H g} g H$. A variação da pressão atmosférica é:

$\Delta P=\rho_{H g} g \Delta H$

Para medir a pressão atmosférica usando a densidade do próprio ar, é preciso conhecer o peso de todo o ar sobre a área considerada. O comprimento $l$ é o tamanho da camada atmosférica, $c-h_{0}$, em que $c$ é a altitude da atmosfera em relação a $h_{0}$ (mais sobre a espessura da atmosfera será considerado adiante). Se $\bar{\rho}_{a r}$ for a densidade média de todo ar acima de $h_{0}$, então a pressão atmosférica é $P_{0}=\bar{\rho}_{a r} g\left(c-h_{0}\right)$. A rigor, a densidade média do ar acima de $h_{0}$ (i.e., com comprimento infinito) difere da densidade do ar limitado ao intervalo finito com comprimento $c-h_{0}$. Entretanto, esta equação será usada mais adiante apenas em relação a uma camada de atmosfera com início e fim conhecidos. Analogamente, numa altitude $h$ não muito distante de $h_{0}$, a pressão atmosférica é $P \approx \bar{\rho}_{a r} g(c-h)$. A variação da pressão atmosférica é:

$\Delta P \approx-\bar{\rho}_{a r} g \Delta h$

Diz-se que $h$ não está muito distante de $h_{0}$ se, dentro do limite de precisão usado, a densidade do ar não variar entre estas duas altitudes. Assim, $\rho_{H g} \Delta H \approx-\bar{\rho}_{a r} \Delta h$. A diminuição de $\Delta H$ com o aumento de $\Delta h$ é consequência de um princípio físico, o equilíbrio hidrostático.

A Equação 2a é exata na medida em que a densidade do mercúrio não muda com a pressão atmosférica. A Equação $2 b$ é uma aproximação, cuja precisão melhora na medida em que $\Delta h$ diminui. No limite quando $\Delta h$ tende a zero, a Equação $2 \mathrm{~b}$ torna-se exata. A densidade em uma camada infinitesimal numa altitude arbitrária já não é mais um valor médio, mas sim uma função de $h$. Isto se representa na forma diferencial:

$d P=-\rho_{a r} g d h$

A relação entre densidade do ar e pressão atmosférica vem da equação do gás ideal: 
$P=\frac{\rho_{a r}}{M} R T$

$R$ é a constante dos gases, $T$ a temperatura termodinâmica e $M$ a massa molar do ar. Isolando $\rho_{a r}$ da Equação 3, substituindo-o na Equação 2c e integrando com os limites correspondentes obtém-se:

$\int_{P_{0}}^{P} \frac{d P}{P}=\frac{-1}{R} \int_{0}^{h} \frac{M}{T} g d h$

Se a massa molar, a aceleração da gravidade e a temperatura forem independentes da altura, o resultado da integração é a Equação 1, porque:

$\int_{P_{0}}^{P} \frac{d P}{P}=\frac{-M g}{R T} \int_{0}^{h} d h$

Castellan ${ }^{10}$ considera as pressões, $P$ e $P+d P$, em duas altitudes, $h$ e $h+d h$. Determina a massa de ar acima de $h, m$, e a massa de ar acima de $h+d h, m^{\prime}$. A massa de ar entre $h$ e $h+d h$ é $d m=m-m^{\prime}$. Então, $P+d P=\frac{(m-d m) g}{A}=\frac{m g}{A}-\frac{g d m}{A}$. Com $d m=\rho A d h$, chega-se à Equação 2c e a dedução prossegue como mostrado acima.

Kikoin e Kikoin ${ }^{11}$ argumentam que a pressão muda de $P$ para $P-d P$, quando a altitude passa de $h$ e $h+d h$. Isto é uma forma artificial de forçar o sinal negativo na diferencial da Equação 2.

Historicamente, a fórmula barométrica foi deduzida por considerações da hidrostática ${ }^{6}$. A pressão em uma coluna com altura $H$ contendo um gás com comportamento ideal apresenta pressão, numa determinada altura, $z$, igual a $P(z)=P(H)+M(z) g$, se $z<H$. $M(z)$ é a massa do gás por unidade de área desde a altura $z$ até $H: M(z)=\int_{z}^{H} \rho(u) d u$. Usa-se a Equação 3, para obter a equação $P(z)=P(H)+M / R \int_{z}^{H} \frac{g P(u)}{T} d u$, cuja solução é a Equação 1, desde que $g$ e $T$ sejam independentes da altura.

Pantellini ${ }^{1}$ simulou a atmosfera com partículas puntiformes em queda livre e usando uma aproximação unidimensional, tal que a posição de cada partícula fosse completamente especificada na altitude z. Partículas que atingissem o fundo refletir-se-iam elasticamente. Para não violar a hipótese ergódica de Boltzmann, a simulação usa velocidade tridimensional na descrição do movimento essencialmente unidimensional. A hipótese da temperatura constante é plausível, caso o livre caminho médio seja pequeno comparado com a escala de variação das quantidades microscópicas. Segue-se que se a distribuição de velocidades é maxwelliana em uma dada altura, ela tem de ser maxwelliana com a mesma temperatura, qualquer que seja a altura. $\mathrm{O}$ resultado é a diminuição exponencial da pressão de equilíbrio com a altitude.

Por fim, a fórmula barométrica foi, segundo Pantellini, abordada também por Becker. ${ }^{12}$ Baseia-se na pressuposição de que o número de partículas em um volume num dado instante é igual ao número de partículas em um instante seguinte. Impondo-se a restrição do campo gravitacional constante e as equações de movimento, obtémse a fórmula barométrica.

\section{Parte II: Extensão da fórmula barométrica}

A passagem na Equação 4 não é mera matemática; é consequência de realidade física. É aparentemente trivial, entretanto, dá origem a uma rica discussão. Até que ponto é admissível assumir a independência da temperatura, da aceleração da gravidade e da massa molar com a altura? Para responder, é preciso investigar, um a um, como esses parâmetros são influenciados pela altitude.

\section{Modelo II - Efeito da temperatura}

Supondo que a temperatura varie linearmente com a altitude, tem-se:

$T=T_{0}+a h$

O parâmetro $a$ significa a variação de temperatura com a elevação de um metro, expresso em $\mathrm{K} / \mathrm{m}$; pode ser negativo ou positivo. $\mathrm{O}$ parâmetro $T_{0}$ significa a temperatura na altitude de referência. $T_{0}$ e $a$ são independentes da altura. Substituindo a Equação 5 na Equação 4a, se a massa molar e a aceleração da gravidade forem independentes da altura, obtém-se após integração:

$P=P_{0}\left(\frac{a h+T_{0}}{T_{0}}\right)^{\frac{-M g}{a R}}$

O termo $a$ não pode ser nulo porque o expoente torna-se indeterminado. Assim, a análise do comportamento de $P$ com o aumento da altura depende somente do sinal de $a$. Como a temperatura termodinâmica é sempre positiva, o termo entre parênteses é maior ou menor que a unidade, respectivamente, se $a$ for positivo ou negativo. Se $a$ for positivo, o expoente é negativo. Assim, $P$ diminui com a altitude. Se $a$ for negativo, o expoente é positivo. $\mathrm{O}$ termo entre parêntese, fracionário, elevado a um número positivo, faz também com que o resultado, $P$, diminua com a altitude.

É interessante notar que a altura não aparece no expoente da Equação 6. Reintroduzindo a Equação 5 na Equação 6, obtémse uma equação na forma $P=P_{0}\left(T / T_{0}\right)^{k}$, em que $k$ é constante. Verkley e Gerkema9 obtiveram esta equação através de um modelo termodinâmico. Maximizaram a entropia de uma coluna de gás seco com massa constante e consideraram o movimento molecular desprezível em comparação com o movimento de convecção. Concluíram que a coluna de gás é isoentrópica. Portanto, a contraposição entre o modelo I e o modelo II é que o primeiro é isotérmico e o segundo é isoentrópico. A única diferença entre o modelo I e II está na Equação 5. Assim, o perfil de temperatura determina o perfil de entropia numa coluna de gás sujeita a um campo gravitacional constante.

\section{Modelo III - Efeito da aceleração da gravidade}

Pode a fórmula barométrica ser explorada para investigar a atmosfera na altitude dos satélites comerciais? Este tipo de questão leva a investigar o efeito da aceleração da gravidade. A resposta vem da equação da gravitação, que exprime a força de atração, $F$, entre dois corpos de massas $m_{1}$ e $m_{2}$ distantes entre si por uma distância $x$ :

$F=G \frac{m_{1} m_{2}}{x^{2}}$

O valor da gravidade pode ser calculado assumindo a Terra como uma esfera, cujo centro de massa esteja localizado no seu centro. Segunda a referência 13, a constante gravitacional, $G$, é $6,67259 \times$ $10^{-11} \mathrm{~N} \mathrm{~m}^{2} \mathrm{~kg}^{-2}$; a massa da Terra, $M_{T}, 5,98 \times 10^{24} \mathrm{~kg}$; seu raio, $R_{T}$, $6,378136 \times 10^{6} \mathrm{~m}$ (ver referência 14 sobre valores autoconsistentes das constantes universais). Assim, um corpo na altura $h$ em relação ao nível do mar está distante do centro da Terra em $(R+h)$ metros. $\mathrm{O}$ peso de uma massa qualquer, $m_{2}$, é $m_{2} g$. A força peso é a força gravitacional, $m_{2} g=G \frac{M_{T} m_{2}}{\left(R_{T}+h\right)^{2}} \cdot$ A aceleração da gravidade terrestre é então dada por: 
$g=\frac{G M_{T}}{\left(R_{T}+h\right)^{2}}$

$\frac{g}{\left(\mathrm{~m} \mathrm{~s}^{-2}\right)}=\frac{3,99 \times 10^{14}}{\left(6,378136 \times 10^{6}+h^{2}\right)}$

Substituindo-se a Equação 8 na Equação 4a, assumindo $M, T$ e $g$ constantes, obtém-se após integração:

$P=P_{0} e^{\frac{M}{R T}\left(\frac{G M_{T}}{R_{T}+h}-R_{T}\right)}$

$P=P_{0} e^{\left(\frac{-720,19 h}{6,378136 \times 10^{6}+h}\right)}$

\section{Modelo IV - Efeito da aceleração da gravidade e da temperatura}

Este caso resolve-se inserindo a Equação 8 a e a Equação 5 na Equação $4 \mathrm{a}$, assumindo que a massa molar seja independente da altura:

$\int_{P_{0}}^{P} \frac{d P}{P}=\frac{-G M}{R} \int_{0}^{h} \frac{1}{\left(T_{0}+a h\right)} \frac{M_{T}}{\left(R_{T}+h\right)^{2}} d h$

A integração $0^{15}$ da Equação 10 fornece:

$\ln \frac{P}{P_{0}}=\frac{G M M_{T}}{R\left(a R_{T}-T_{0}\right)} \frac{1}{\left(R_{T}+h\right)}+$

$\frac{G M M_{T} a}{R\left(T_{0}-a R_{T}\right)^{2}} \ln \left[\left(\frac{R_{T}}{R_{T}+h}\right)\left(\frac{T_{0}+a h}{T_{0}}\right)\right]+\frac{G M\left(M_{T} T_{0} / R_{T}-M_{T} a\right)}{R\left(T_{0}-a R_{T}\right)^{2}}$

Substituindo as constantes conhecidas e usando $a=-6 / 885 \mathrm{~K} \mathrm{~m}^{-1}$ (vide infra, Comparação entre Modelo I e o Modelo II), a pressão atmosférica terrestre, no sistema internacional de unidades é:

$\ln \frac{P}{P_{0}}=\frac{3,19631 \times 10^{7}}{\left(6,378136 \times 10^{6}+h\right)^{+}}$

$4,97649 \ln \frac{303-\frac{2 h}{295}}{6,378136 \times 10^{6}+h}+44,5279$

\section{Modelo V - Efeito da massa molar}

A massa molar do ar oscila entre 28,9 e 29,2 g/mol (vide infra, Tabela 1). Isto é insignificante no presente contexto. Se, em outro contexto, por exemplo, em atmosfera de planetas muito diferentes da nossa atmosfera, o efeito for significativo, o melhor a fazer é estimar a massa molar média, $\bar{M}$, usando o teorema do valor médio, ${ }^{16}$

$\bar{M}=\frac{1}{h} \int_{0}^{h} M d h$.

\section{Parte III: A atmosfera-padrão}

A atmosfera-padrão ${ }^{17}$ é uma representação idealizada das condições consideradas típicas em latitudes médias. Foi desenvolvida pela necessidade de uma atmosfera de referência para uso no projeto de aeronaves e seus instrumentos. Ela é definida com temperatura de $288,15 \mathrm{~K}$ e pressão 1013,25 hPa no nível do mar com taxas de variação que mudam conforme a altitude. A Tabela 1 mostra suas características. A atmosfera ${ }^{18}$ é mais complexa, mas a descrição simplificada é suficiente na discussão dos resultados.

\section{RESULTADOS E DISCUSSÃO}

\section{Aplicações elementares do modelo I}

Existem no mercado barômetros tão sensíveis que, além de mostrarem a pressão atmosférica, convertem a informação barométrica em informação hipsométrica, ou seja, determinam a variação da altitude. O teste mais simples é subir (ou descer) uma escadaria com altura entre degraus conhecida e verificar se a altura é compatível com a diferença de altitude calculada. Assim, em um dia quente $\left(31^{\circ} \mathrm{C}\right)$, a altitude de $841 \mathrm{~m}$ no pé de uma escada externa de um dos edifícios da universidade medida em um barômetro/altímetro digital (Oregon ${ }^{\circledR}$, modelo EB833) passou para $845 \mathrm{~m}$ vinte degraus acima. O barômetro fora previamente calibrado na estação de observação meteorológica do Instituto de Geografia, no mesmo campus. A altura de cada degrau era $20 \mathrm{~cm}$. A altitude passou para $848 \mathrm{~m}$ com outros vinte degraus acima. Portanto, a altura entre o primeiro e o quadragésimo degrau é 7,0 m. A medida de pressão não variou $(930 \mathrm{hPa})$. A Equação 1 mostra que o aumento de $7,0 \mathrm{~m}$ leva a um abaixamento de pressão de $P_{0} e^{\frac{-M g}{R T} h}$, ou seja, $930 e^{\frac{-29,0 \times 10^{-3} \times 9,79 \times 7,0}{8,314 \times(31+273)}}$, que vale $929,3 \mathrm{hPa}$. A diferença (700 Pa) não é suficiente para ser detectada no visor, mas internamente o barômetro tem sensibilidade suficiente para lidar com tais pequenas variações.

Em sala de aula, há muito ainda que explorar: o cuidado necessário com unidades; a importância do domínio do uso das calculadoras científicas; o uso de programas gráficos para ilustrar o efeito dos vários parâmetros no perfil do gráfico da pressão em função da altitude; o uso da imprensa, que frequentemente noticia ${ }^{19}$ relatos de expedições a altas montanhas em que inevitavelmente se menciona a escassez

Tabela 1. Características da atmosfera-padrão, conforme referência 17. Massa molar calculada a partir da Equação 3

\begin{tabular}{|c|c|c|c|c|c|}
\hline Altitude / km & $\mathrm{T} / \mathrm{K}$ & $\mathrm{a} / \mathrm{K} \mathrm{km}^{-1}$ & $\mathrm{P} / \mathrm{hPa}$ & $\rho / \mathrm{kg} \mathrm{m}^{-3}$ & $\mathrm{M} / \mathrm{g} \mathrm{mol}^{-1}$ \\
\hline 0 & 288,15 & $-6,5$ & 1013,25 & 1,225 & 28,96 \\
\hline 11 & 216,65 & 0,0 & 226,3206 & 0,364 & 28,97 \\
\hline 20 & 216,65 & 1,0 & 54,7489 & $8,803 \times 10^{-2}$ & 28,96 \\
\hline 32 & 228,65 & 2,8 & 8,6802 & $1,322 \times 10^{-2}$ & 28,95 \\
\hline 47 & 270,65 & 0,0 & 1,1091 & $1,428 \times 10^{-3}$ & 28,97 \\
\hline 51 & 270,65 & $-2,8$ & 0,6694 & $8,616 \times 10^{-4}$ & 28,96 \\
\hline 71 & 214,65 & $-2,0$ & 0,0396 & $6,421 \times 10^{-5}$ & 28,94 \\
\hline 84,852 & 186,95 & - & 0,0037 & $6,958 \times 10^{-6}$ & 29,23 \\
\hline
\end{tabular}


de oxigênio e a dificuldade em se respirar nessas altitudes; a relação entre a Equação 1 com a pressão parcial ${ }^{10}$ e suas consequências.

\section{Comparação entre o modelo I e o modelo II}

Usando o modelo I, calculou-se a pressão em Canudos, BA, conhecendo-se a pressão na cidade litorânea de Aracajú, SE. Canudos fica $400 \mathrm{~m}$ acima do nível do mar. Calculou-se a massa molar média do ar igual a 29,0 g/mol (média ponderada considerando o ar atmosférico como $\mathrm{N}_{2}(78 \%), \mathrm{O}_{2}(21 \%), \mathrm{CO}_{2}(0,5 \%)$ e $\left.\mathrm{Ar}(0,5 \%)\right)$. Admitiu-se que a temperatura fosse constante, igual a $30,0{ }^{\circ} \mathrm{C}$, e aceleração da gravidade de $9,80 \mathrm{~m} / \mathrm{s}^{2}$. O resultado é que a pressão em Canudos é 95,6\% da pressão de Aracaju. Este resultado está sujeito às restrições mencionadas anteriormente, mas ilustra o alcance da equação.

O refinamento do resultado obtido se fez através do modelo II, Equação 6. Supondo que Canudos esteja a $38,0{ }^{\circ} \mathrm{C}$, como é comum nas regiões do Sertão, então há uma diferença de $8 \mathrm{~K}$ em $400 \mathrm{~m}$. O parâmetro é então: $a=\frac{8}{400} \mathrm{~K} \mathrm{~m}^{-1}=0,0200 \mathrm{~K} \mathrm{~m}^{-1}$, conquanto que Aracaju se mantenha a $30{ }^{\circ} \mathrm{C}\left(T_{0}=303 \mathrm{~K}\right)$. O resultado é a pressão em Canudos igual a 95,8\% da pressão em Aracaju. Apenas 0,2\% a mais do que o calculado pelo modelo I. Isto ilustra o sucesso da fórmula barométrica.

A próxima etapa é repetir o confronto em situação mais drástica. Sejam colocados os parâmetros do Monte Everest (8850 m, temperatura de $-30{ }^{\circ} \mathrm{C}$ ) como comparação, mantendo Aracaju como referência. O parâmetro $a$ é então: $\frac{(-30+273)-(30+273)}{8850}=\frac{-6}{885} \mathrm{~K} / \mathrm{m}$, bastante próximo ao valor de $a$ da camada mais baixa da atmosfera mostrada na Tabela $1(-6,5 \mathrm{~K} / \mathrm{km})$. Os resultados estão na Tabela 2.

Tabela 2. Predição da pressão atmosférica no Monte Everest, usando diferentes temperaturas. Parâmetros usados: $a=\frac{-6}{885} \mathrm{~K} / \mathrm{m} ; T_{0}=$ $30{ }^{\circ} \mathrm{C}(303 \mathrm{~K}) ; g=9,80 \mathrm{~m} / \mathrm{s}^{2} ; h=8850 \mathrm{~m} ; M=29,0 \times 10^{-3} \mathrm{~kg} / \mathrm{mol}$

\begin{tabular}{lccc}
\hline $100 \times P / P_{0}$ & Temperatura & $a / \mathrm{K} \mathrm{m}^{-1}$ & Modelo \\
\hline 36,8 & $T=+30{ }^{\circ} \mathrm{C}$ & - & I, Eq. (1) \\
28,8 & $T=-30{ }^{\circ} \mathrm{C}$ & - & I, Eq. (1) \\
32,7 & $T_{0}=+30{ }^{\circ} \mathrm{C}$ & $-6 / 885$ & II, Eq. (6) \\
\hline
\end{tabular}

A diferença entre os dois modelos, agora, é $\pm 4 \%$. Em muitas situações, tal erro ainda é aceitável. Este é um dos motivos porque a fórmula barométrica é mais conhecida que o modelo II.

Uma questão importante é sobre a espessura da atmosfera, cujo valor não se pode colocar de forma definitiva. A espessura da atmosfera é estimada desde $5000 \mathrm{~m},{ }^{20} 8500 \mathrm{~m},{ }^{6}$ ou $32 \mathrm{~km} \cdot{ }^{13,21}$ Estende-se para mais de $400 \mathrm{~km}$, parcialmente ionizada, muito além do limite da atmosfera-padrão; mesmo com pressão igual a uma fração de pascal, determina a propagação de ondas de rádio. ${ }^{22}$ Assim, a espessura da atmosfera depende da técnica de medida e da aplicação. Os modelos descrevem a pressão com equações assintóticas. Algum tipo de critério é necessário para estabelecer um limite finito, por exemplo, impor que a pressão seja um percentual da pressão no nível do mar. Este percentual é arbitrário. Escolheu-se aqui a razão $P / P_{0}$ calculada a partir da Tabela 1. Esta razão foi usada nas Equações 1 e 6. A espessura obtida foi comparada à altitude correspondente da atmosfera-padrão (Tabela 3).

O modelo I erra sempre de 20 a $30 \%$, enquanto que o modelo II erra menos de $15 \%$ até altitude de cerca de $30 \mathrm{~km}$. Portanto, a correção da temperatura é um avanço até nestas altitudes. Entretanto, isto não significa que o modelo I substitua o modelo II. A atmosfera apresenta camadas em que a temperatura é constante e apresenta camadas em que a temperatura é variável (ver Tabela 1, parâmetro a). Os modelos I e II descrevem exatamente cada caso. Analisada detalhadamente, a estrutura da atmosfera-padrão recai em última instância nos modelos I e II, mostrando que estes dois modelos não são mutuamente exclusivos.

Tabela 3. Predição da espessura da atmosfera terrestre pelos modelos I e II, comparada com a atmosfera-padrão

\begin{tabular}{lcccc}
\hline $\begin{array}{l}\text { Critério } \\
P / P_{0}\end{array}$ & $\begin{array}{c}h / \mathrm{km} \\
(\text { Tabela } 1)\end{array}$ & $\begin{array}{c}\text { Modelo } \\
\text { I: Eq. }(1) \\
\text { II: Eq. }(6)\end{array}$ & $c / \mathrm{m}$ & $\begin{array}{c}\text { Erro } \\
\text { relativo } \%\end{array}$ \\
\hline \multirow{2}{*}{0,223} & 11 & I & 13289 & 20,8 \\
& & II & 11496 & 4,20 \\
0,0540 & \multirow{2}{*}{20} & I & 25872 & 29,4 \\
& & II & 19641 & $-1,80$ \\
$8,56 \times 10^{-3}$ & \multirow{2}{*}{32} & I & 42198 & 31,9 \\
& & II & 27307 & $-14,7$ \\
$1,09 \times 10^{-3}$ & 47 & I & 60466 & 28,7 \\
& & II & 33140 & $-29,5$ \\
$6,60 \times 10^{-4}$ & \multirow{2}{*}{51} & I & 64913 & 27,3 \\
& & II & 34216 & $-32,9$ \\
$3,91 \times 10^{-5}$ & \multirow{2}{*}{71} & I & 89964 & 26,7 \\
& & II & 38722 & $-45,5$ \\
$3,65 \times 10^{-6}$ & \multirow{2}{*}{84,852} & I & 110984 & 30,8 \\
\hline
\end{tabular}

\section{Análise do modelo III}

Os resultados numéricos do modelo III pouco diferem dos resultados numéricos do modelo I. A Figura 1 compara ambos os modelos com $h$ no intervalo de 40 a $60 \mathrm{~km}$. Este intervalo foi escolhido para tornar a diferença perceptível graficamente. A diferença é insignificante tanto na camada mais baixa quanto na camada mais alta da atmosfera. As duas curvas são sobreponíveis se o gráfico for construído em escala mais ampla (vide infra, Figura 2). Portanto, salvo em aplicações específicas, ${ }^{23}$ é admissível negligenciar a variação de $g$ com a altura e usar o valor de $g$ da superfície.

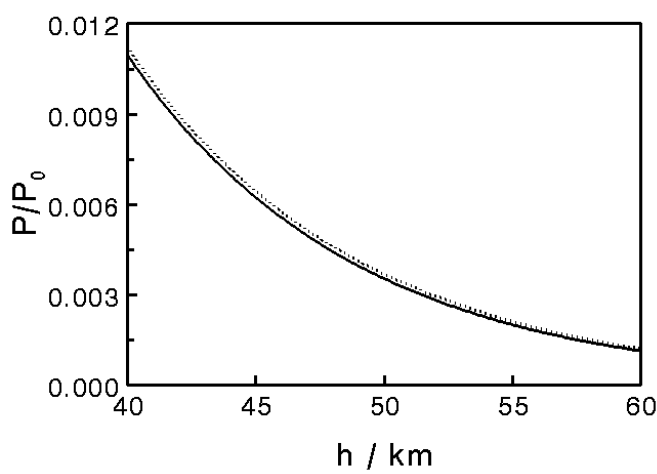

Figura 1. Pressão atmosférica relativa em função da altitude. $T=303 \mathrm{Ke}$ $M=29,0 \mathrm{~g} / \mathrm{mol}$ em ambos os casos. Linha pontilhada: modelo $\mathrm{I} ; \mathrm{g}=9,80 \mathrm{~m} /$ $s^{2}$; linha contínua: modelo III, g variável conforme Equação 8 


\section{Modelo IV}

A Figura 2 reúne a predição da atmosfera terrestre com os modelos I a IV. Na escala apresentada, das quatro curvas, somente duas curvas são distinguíveis: a curva superior corresponde aos modelos I e III, discutido na seção anterior. A curva inferior corresponde aos modelos II e IV. A sobreposição dos modelos II e IV mostra que a variação da temperatura é muito mais importante que a variação da aceleração da gravidade. Tal como o caso anterior, é admissível negligenciar a variação de $g$.

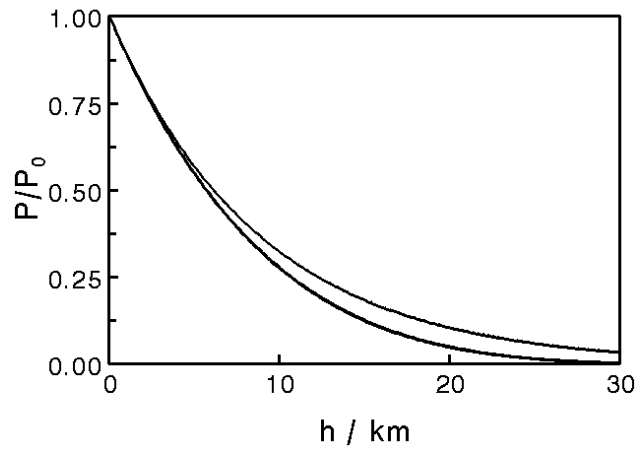

Figura 2. Comparação das predições obtidas com os quatro primeiros modelos. Curva superior: modelos I e III, indistinguíveis; curva inferior: modelos II e IV, indistinguíveis. Parâmetros usados: $M=29,0 \mathrm{~g} / \mathrm{mol} . \mathrm{g}=9,80 \mathrm{~m} / \mathrm{s}^{2} ; \mathrm{g}$ variável nos modelos III e IV conforme Equação $8 b . T=303 \mathrm{~K}$; T variável nos modelos I e II ( $\left.T_{0}=303 \mathrm{~K}, a=-6 / 885 \mathrm{~K} / \mathrm{m}\right)$

\section{Modelo V}

Em vista da variação insignificante da massa molar com a altitude, associada ao conhecimento, ainda que simplificado, da estrutura da atmosfera terrestre através do modelo da atmosfera-padrão (Tabela 1), é correto retirar a massa molar do integrando na Equação 4, assumindo-a constante.

\section{Verificação das predições com resultados experimentais}

Da discussão anterior, decorre que a comparação mais importante é entre os modelos I e II. Para aplicar os fundamentos teóricos aos dados experimentais, usaram-se medidas de pressão atmosférica e de temperatura registradas em plataformas de coleta de dados do Instituto Nacional de Pesquisas Espaciais (INPE). Foram escolhidas sete cidades em que tais plataformas estavam ativas no período arbitrário de primeiro a doze de setembro de 2008. O INPE monitorou a temperatura e a pressão de $3 \mathrm{em} 3 \mathrm{~h}$, totalizando oito medições por dia em cada localidade. Os dados ${ }^{24}$ foram copiados, a média aritmética foi calculada e compilada na Tabela 4.

Tabela 4. Média aritmética da temperatura e da pressão atmosférica em algumas localidades brasileiras no período de primeiro a doze de setembro de 2008, calculadas a partir de dados originais do INPE

\begin{tabular}{lccc}
\hline Cidade & Altitude/m & Temperatura/ ${ }^{\circ} \mathrm{C}$ & $\begin{array}{c}\text { Pressão } \\
\text { atmosférica/ } \\
\text { hPa }\end{array}$ \\
\hline $\begin{array}{l}\text { Caraguatatuba, } \\
\text { SP }\end{array}$ & 3 & 21,3 & 1022 \\
$\begin{array}{l}\text { Recife, PE } \\
\begin{array}{l}\text { Fernando de } \\
\text { Noronha, PE }\end{array}\end{array}$ & 10 & 25,5 & 1015 \\
$\begin{array}{l}\text { Porto } \\
\text { Trombetas, PA }\end{array}$ & 60 & 26,5 & 1006 \\
$\begin{array}{l}\text { Salvador, BA } \\
\begin{array}{l}\text { Quixeramobim, } \\
\text { CE }\end{array}\end{array}$ & 85 & 27,0 & 934,6 \\
$\begin{array}{l}\text { Barretos, SP } \\
\text { Meruoca, CE }\end{array}$ & 549 & 24,3 & 1016 \\
\hline
\end{tabular}

Os parâmetros da Tabela 4 foram usados nas Equações 1 e 6. Caraguatatuba foi eleita como referência, tendo como único critério sua altitude mais baixa. Os resultados estão na Tabela 5.

O erro aumenta conforme aumenta a altitude em ambos os modelos. O modelo I erra mais que o modelo II.

Mesmo no nível do mar, a pressão atmosférica pode variar no plano geodésico. A Figura 3 mostra as linhas ao longo das quais a pressão é constante. ${ }^{25}$ Pressões desiguais em diferentes pontos no espaço representam gradientes de pressão. Gradientes de pressão originam ventos e alteram a pressão atmosférica nas suas vizinhanças. Assim, fica claro que o nível do mar não pode ser usado como pressão-padrão (daí a IUPAC ${ }^{26}$ ter recomendado uma nova unidade de

Tabela 5. Comparação entre predição e medidas experimentais da pressão atmosférica em diferentes localidades. Parâmetros usados: $g=9,80$ $\mathrm{m} \mathrm{s}^{-2} ; M=0,0290 \mathrm{~kg} / \mathrm{mol}$; média entre $21,3{ }^{\circ} \mathrm{C}$ e $T$ (ambos expressos em Kelvin) no modelo I; $a=\frac{T-21,3}{h-3}$, ( $T$ aqui expresso em graus Celsius) no modelo II. Valores de $T$ e $h$ extraídos da Tabela 4

\begin{tabular}{|c|c|c|c|c|c|}
\hline \multirow{2}{*}{$\begin{array}{l}\text { Localidade } \\
-\end{array}$} & \multicolumn{3}{|c|}{ Pressão atmosférica } & \multicolumn{2}{|c|}{ Diferença relativa } \\
\hline & Mod. (I) & Mod. (II) & INPE & Mod. (I) & Mod. (II) \\
\hline Caraguatatuba, SP & 1022 & 1022 & 1022 & - & - \\
\hline Recife, PE & 1021 & 1021 & 1015 & $0,6 \%$ & $0,6 \%$ \\
\hline $\begin{array}{l}\text { Fernando de Noronha, } \\
\text { PE }\end{array}$ & 1019 & 1018 & 1006 & $1,3 \%$ & $1,2 \%$ \\
\hline Porto Trombetas, PA & 1017 & 1015 & 934,6 & $8,8 \%$ & $8,6 \%$ \\
\hline Salvador, BA & 1015 & 1012 & 1016 & $-0,1 \%$ & $-0,4 \%$ \\
\hline Quixeramobim, CE & 1007 & 1000 & 986,3 & $2,1 \%$ & $1,4 \%$ \\
\hline Barretos, SP & 978,4 & 959,4 & 948,0 & $3,2 \%$ & $1,2 \%$ \\
\hline Meruoca, CE & 968,9 & 945,9 & 919,6 & $5,4 \%$ & $2,9 \%$ \\
\hline
\end{tabular}


pressão, o bar, em destituição da atm). De forma estrita, os modelos descritos deveriam ser aplicados a uma coluna de ar acima de uma localidade. A despeito destas considerações, a maior discordância na Tabela 5 é inferior a $9 \%$.

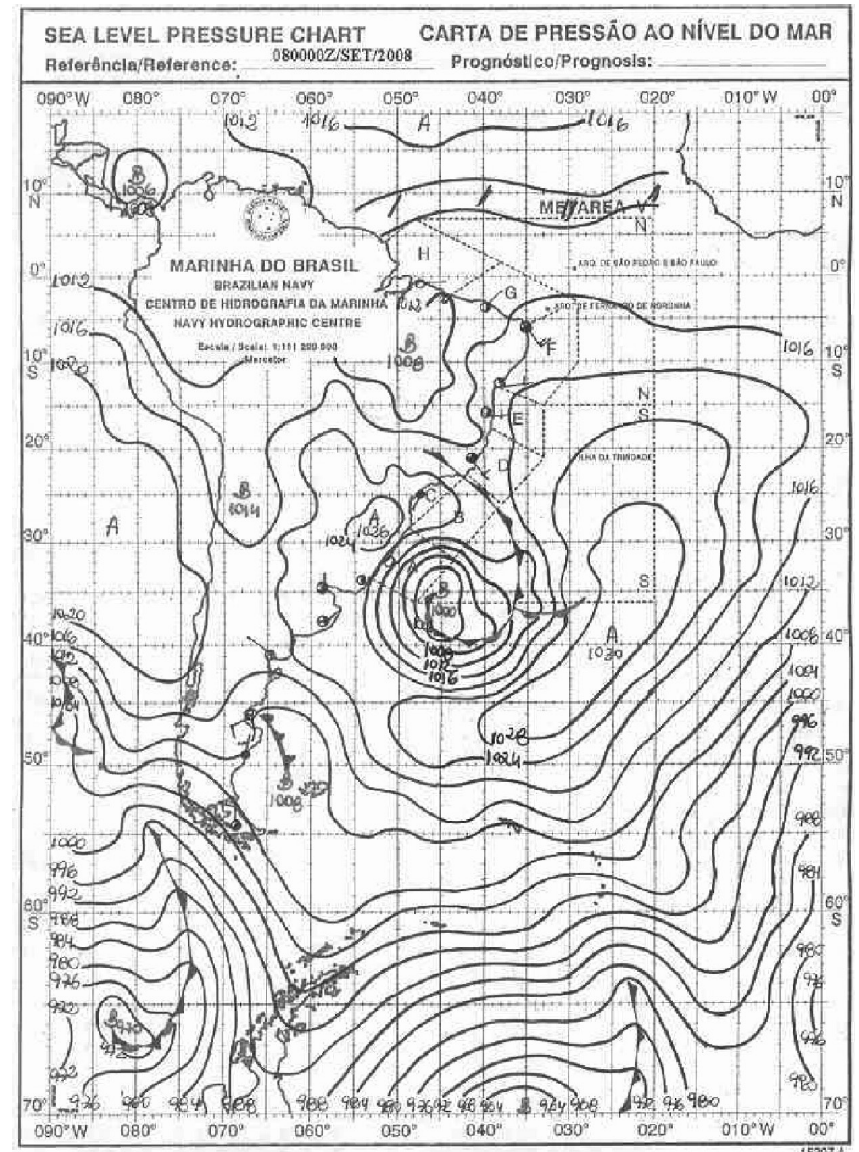

Figura 3. Carta sinótica mostrando as linhas isobáricas do Oceano Atlântico sul e parte do Oceano Pacífico no dia 8 de setembro de 2008 às 00h00min (horário universal). A pressão atmosférica no nível do mar variava de 964 hPa (nas imediações do Círculo Ártico) a 1030 hPa (na coordenada geográfica aproximada de $25^{\circ}$ oeste, $40^{\circ}$ sul). Copyright (C) 2008, 2009, Marinha Brasileira, reproduzida com permissão

\section{CONCLUSÃO}

A fórmula barométrica proporciona oportunidades para discutir temas diferentes de modo interrelacionado. O modelo II confere considerável avanço em relação à precisão. Os modelos I e II fundamentam o modelo da atmosfera-padrão. As predições são significativamente boas, embora seja questionável aplicar os modelos apresentados à atmosfera, que não está em equilíbrio.

\section{AGRADECIMENTOS}

Aos revisores. Suas observações aperfeiçoaram a estrutura do manuscrito e enriqueceram o texto.

\section{REFERÊNCIAS E NOTAS}

1. Pantellini, F. G. E.; Am. J. Phys. 2000, 68, 61.

2. Gould, F. A.; Stephenson R. J.; Encyclopaedia Britannica 1970, 3, 179.

3. Halley, E.; Philos. Tran. R. Soc. 1686, 16, 104; http://www.jstor.org/ pss/101848, acessada em Dezembro 2008.

4. Laplace, P.-S.; Traité de Mecanique Celeste, Hilliard Gray, Little \& Wilkins: Boston, 1798, cap. 4; citado por Berberan-Santos, ref. 6.

5. Coffey, L.; Am. J. Phys. 2006, 74, 757.

6. Berberan-Santos, M. N.; Bodunov, E. N.; Poliani, L.; Am. J. Phys. 1997, $65,404$.

7. Martins, R. de A.; Cad. Hist. Fil. Ciência 1989, 1, 9.

8. Ferreira, R.; Quim. Nova 1991, 14, 125.

9. Verkley, W. T. M.; Gerkema, T.; Am. Meteorol. Soc. 2004, 61, 931; Akmaev, R. A.; Q. J. Meteorol. Soc. 2008, 134, 187, doi:10.1002/qj.209.

10. Castellan, G. C.; Fundamentos de Físico-química, Ed. Livros Técnicos e Científicos: Rio de Janeiro, 1986, cap. 2, p. 24.

11. Kikoin, I.; Kikoin, A.; Física Molecular, Ed. Mir: Moscou, 1971, cap. 1 , seção 7 .

12. Becker, R.; Theorie der Wärme, Springer-Verlag, 1978.

13. Linde, D. R., ed.; RC Handbook of Chemistry and Physics, $86^{\text {th }}$ ed., Taylor \& Francis Group: New York, 2006, cap. 14.

14. Mohr, P. J.; Taylor, B. N.; Newell, D. B.; J. Phys. Chem. Ref. Data 2008 , 37, 1187; http://physics.nist.gov/cuu/Constants/index.html, acessada em Fevereiro 2009.

15. Tsypkin; A. G.; Tsypkin, G. G.; Fórmulas Matemáticas, Ed. Mir: Moscou, 1990.

16. Tan, S. T.; College Mathematics, PWS-KENT Pub. Co.: Boston, 1988, p. 929.

17. http://mtp.jpl.nasa.gov/notes/altitude/StdAtmos1976.html, acessada em Fevereiro 2009

18. http://dequim.ist.utl.pt/docentes/2219/QF2/docs/Estrutura $\% 20 \mathrm{e} \% 20$ composicao\%20da\%20atmosfera.pdf, acessada em Setembro 2008.

19. http://www.bbc.co.uk/portuguese/reporterbbc/story/2008/08/080804_ k211mortos_ac.shtml, acessada em Setembro 2008.

20. Vollmer, M.; Am. J. Phys. 2003, 71, 979.

21. http://www.pdas.com/atmthick.htm, acessada em Fevereiro 2008.

22. Hutchinson, C., ed.; The ARRL Handbook, $78^{\mathrm{a}}$ ed., ARRL Inc.: Newington, 2000.

23. Telford, W. M.; Geldart, L. P.; Sheriff, R. E.; Applied Geophysics, Cambridge University Press, 1990, cap. 2, seção 3.

24. http://satelite.cptec.inpe.br/PCD/, acessada em Setembro 2008.

25. http://www.mar.mil.br/dhn/chm/meteo/prev/cartas/cartas.htm, acessada em Setembro 2008

26. http://www.iupac.org/goldbook/S05910.pdf, acessada em Setembro 2008. 